\title{
Duty Cycle and Modulation Efficiency of Two-Channel Hadamard Transform Time-of-Flight Mass Spectrometry
}

\author{
Oh Kyu Yoon, Ignacio A. Zuleta, Joel R. Kimmel,* Matthew D. Robbins, \\ and Richard N. Zare \\ Department of Chemistry, Stanford University, Stanford, California, USA
}

\begin{abstract}
Hadamard transform time-of-flight mass spectrometry (HT-TOFMS) is based on the pseudorandom gating of ion packets into a time-of-flight mass-to-charge analyzer. In its typical implementation, the technique is able to monitor continuous ion sources with a $50 \%$ duty cycle, independent of all other figures of merit. Recently, we have demonstrated that the duty cycle can be extended to $100 \%$ using patterned, two-channel detection. Two-channel HTTOFMS involves the simultaneous optimization of paired one-channel experiments and imposes more stringent conditions to achieve high-quality spectra. An ion modulation device, known as Bradbury-Nielson Gate (BNG), is central to HT-TOFMS. It is an ideal deflection plate, capable of transmitting or deflecting an ion beam according to a known binary sequence without changing the times-of-flight of the ions. Analytical equations are derived that accurately describe the ion modulation process of the BNG as confirmed by good agreement with SimIon simulations and ion beam imaging experiments. From these expressions, the duty cycle and ion modulation efficiency were calculated for various BNG parameters, ion beam characteristics, and detector dimensions, which permit the optimum conditions to be chosen for the two-channel experiment. We conclude that the outer detector should be three times the maximum deflection angle to detect all deflected ions (100\% duty cycle) and that the difference between the modulated ion counts in the sequence elements 0 and 1 should be maximized to achieve high modulation efficiency. This condition is best achieved by tight focusing of the ion beam in the center of the inner detector. When both channels are optimized, the two-channel advantage can be exploited to achieve a further improvement over a single-channel experiment. (J Am Soc Mass Spectrom 2005, 16, 1888-1901) (c) 2005 American Society for Mass Spectrometry
\end{abstract}

$\mathrm{H}$ adamard transform time-of-flight mass spectrometry (HT-TOFMS) [1] is based on modulation of a continuous ion beam according to a pseudorandom binary sequence, and it has a constant duty cycle of $50 \%$ independent of all other figures of merit. Recently, we have demonstrated that the duty cycle can be extended to $100 \%$ using patterned, twochannel (2C) detection [2]. From the high duty cycle, the technique has the advantages of high efficiency, increased sensitivity, and/or rapid acquisition of mass spectra when monitoring continuous ion sources compared with conventional on-axis TOFMS. Orthogonal extraction TOFMS [3, 4] is the more widely used method of achieving high duty cycle, but some trade-off must be accepted between mass range covered and duty cycle achieved. Often these trade-offs are acceptable, but some situations might benefit from the ap-

Published online September 29, 2005

Address reprint requests to Professor R. N. Zare, Department of Chemistry, Stanford University, Room 113, S. G. Mudd Bldg., Stanford, CA 94305-5080, USA. E-mail: zare@stanford.edu

* Current address: Cooperative Institute for Research in the Environmental Sciences, University of Colorado, Boulder, CO 80309-0216. proach described here. The 2C-HT-TOFMS involves the simultaneous optimization of paired one-channel (1C) experiments. Detecting high-quality spectra on both channels requires that the ion modulation be welldefined, but the effects of ion modulation on performance have not been discussed in detail.

The principles of HT-TOFMS have been presented in previous publications [1, 2, 5]. The most important component of the HT-TOFMS instrument is an ion modulation device, called the Bradbury-Nielson Gate (BNG) [6, 7]. The BNG is an ideal deflection plate, capable of transmitting or deflecting an ion beam according to a known binary sequence without changing the flight times of the ions. It consists of two finely spaced, interleaved sets of wire electrodes that are electrically isolated from one another and that lie in a plane perpendicular to the trajectory of the ion beam (Figure 1c). When both wire sets are at relative ground (sequence element 1), all ions are transmitted. When the voltage of one wire set is raised to a positive bias as the voltage of the other is lowered to a negative bias of equal magnitude (sequence element 0), all ions are deflected off-axis into two branches. The deflection 


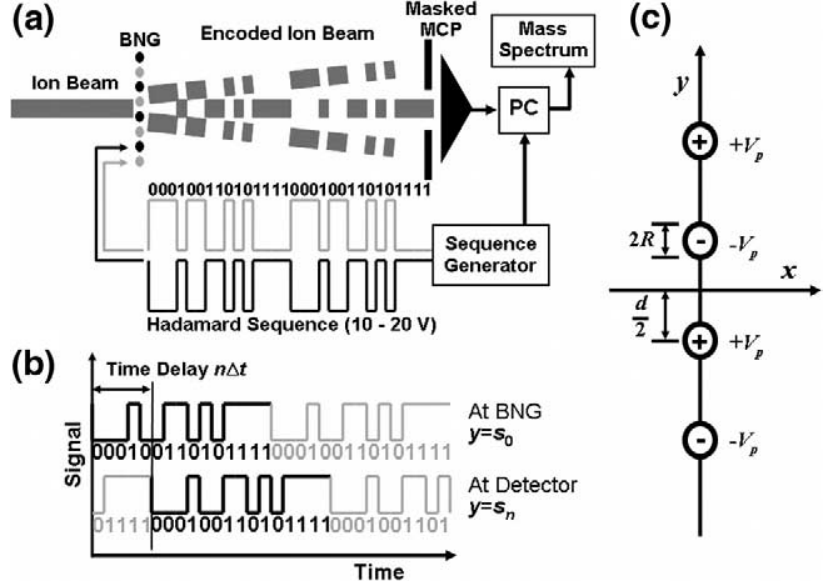

Figure 1. Schematic of (a) the HT-TOFMS, (b) the timing of the BNG and detector signals, and (c) the BNG. The BNG consists of two finely spaced, interleaved sets of wire electrodes, where the wire spacing $d$ and the radius of the wires $R$ are typically 100 and $10 \mu \mathrm{m}$, respectively. A continuous ion beam is modulated by the BNG according to the Hadamard sequence from a sequence generator. The transmitted ion beam is detected by the MCP detector, and the signal is deconvoluted in a personal computer (PC) to obtain the mass spectra. In 2C-HT-TOFMS, the deflected beam is also detected by the outer anode of the MCP detector. The signal at the detector will be the Hadamard sequence with a time delay that depends on the mass of the ion species.

causes the ions introduced into the drift region during sequence element 0 to miss the inner detector.

The schematic of the instrument is in Figure 1a. A continuous, accelerated ion beam is modulated on and off the axis of detection following a Hadamard sequence that is applied at a rate of $10 \mathrm{MHz}$. In 1C-HTTOFMS, deflected ion branches are blocked and only the transmitted ions are detected by the multichannel plate $(\mathrm{MCP})$ detector. The detected signal from the thousands of ion packets is mathematically deconvoluted to recover the time-of-flight mass spectrum. The binary Hadamard sequence employed in this technique is called the maximal length pseudo random sequence (MLPRS) [8]. The sequence has a length of $N=2^{n}-1$, where $n$ is typically $11(N=2047)$, and consists of half $1 \mathrm{~s}$ and half $0 \mathrm{~s}$, which gives the technique its $50 \%$ duty cycle. In 2C-HT-TOFMS, a dual-anode detector is used [2]. The transmitted ions are detected by the inner anode and the deflected ion branches are detected by the outer anode. Each channel has 50\% duty cycle, thus allowing a $100 \%$ duty cycle to be achieved.

The ion beam is modulated at the BNG according to a Hadamard sequence. If the ion current were measured at the BNG, the signal would be the Hadamard sequence scaled by the average intensity. Let's denote the sequence as a length- $N$ column vector $\mathbf{s}_{0}$ (Figure 1b). After modulation, the ions will drift toward the detector in the flight chamber with flight times that depend on the mass of the ions. For a single species with a time delay $n \Delta t$, where $\Delta t$ is the time width of each data acquisition element, the signal at the detector will be the sequence with a time delay $n \Delta t$. This can be denoted as $\mathbf{s}_{n}$, which is the sequence vector $\mathbf{s}_{0}$ that has been cyclically shifted by $n$ places. For multiple ion species with different time delays, the detected signal $\mathbf{y}$ is the sum of all sequences that have different time delays

$$
\mathbf{y}=\sum_{i=0}^{N-1} x_{i} \mathbf{s}_{i}=\left[\begin{array}{llll}
\mathbf{s}_{0} & \mathbf{s}_{1} & \ldots & \mathbf{s}_{N-1}
\end{array}\right]\left[\begin{array}{c}
x_{0} \\
\vdots \\
x_{N-1}
\end{array}\right]=\mathbf{S} \mathbf{x}
$$

where $x_{i}$ is the intensity of the $i^{\text {th }}$ ion species. Thus, $\mathbf{y}$ can be represented as a convolution of the ion modulation matrix $\mathbf{S}$ and the intensity vector $\mathbf{x}$. The matrix $\mathbf{S}$ is an $N \times N$ right-circulant matrix [8], where the first column is the sequence $\mathbf{s}_{0}$, and the other columns are the cyclically shifted vectors $\mathbf{s}_{1}$ through $\mathbf{S}_{\mathrm{N}-1}$.

The deconvolution matrix $\mathbf{R}$ is given by

$$
\mathbf{R}=\left[\begin{array}{c}
\mathrm{w}_{0} \\
\mathrm{w}_{1} \\
\vdots \\
\mathrm{w}_{N-1}
\end{array}\right]=\frac{N+1}{2} \mathbf{S}^{-1}
$$

where $\mathrm{w}_{i}$ is a length- $N$ row vector derived from the transpose of $\mathbf{s}_{i}$ by changing all 0 s to -1 s. The matrix $\mathbf{R}$ is the inverse of the matrix $\mathbf{S}$ scaled by $(N+1) / 2$. This factor is necessary because all ion counts in the detected signal $\mathbf{y}$ result from real ions and the peak intensities after deconvolution should represent the actual number of measured ions. Thus, in the absence of noise, the deconvoluted mass spectrum vector $\mathbf{z}$ is

$$
\mathbf{z}=\mathbf{R} \mathbf{y}=\left(\frac{N+1}{2} \mathbf{S}^{-1}\right)(\mathbf{S} \mathbf{x})=\frac{N+1}{2} \mathbf{x}
$$

which shows the expected $N / 2$ increase in signal intensity.

In the current setup of HT-TOFMS, which uses an electrospray ion source, the ion current is dominated by shot noise, which depends on the signal intensity. Under shot-noise conditions, we have shown recently [5] that the multiplexing advantage of HT-TOFMS in peak height precision (PHP) compared to what we call on-axis TOF is given by

$$
\frac{\mathrm{PHP}^{\mathrm{HT}}}{\mathrm{PHP}^{\mathrm{TOF}}}=\sqrt{\frac{f_{\mathrm{HT}}}{f_{\mathrm{TOF}}}} F_{i}=\sqrt{\frac{N}{2} F_{i}}
$$

where $F_{i}$ is the relative ion population of the peak of interest, and $f_{\mathrm{HT}}$ and $f_{\mathrm{TOF}}$ are the duty cycles of HTTOFMS and on-axis TOFMS, respectively $\left(f_{\mathrm{HT}}=(\mathrm{N}+\right.$ $\left.1) / 2 \mathrm{~N} \approx 50 \%, f_{\text {TOF }}=1 / N\right)$. The PHP is defined as the peak height divided by its standard deviation. This figure of merit is different from the signal-to-noise ratio (SNR), which is defined as the peak height divided by the standard deviation of the baseline. In HT-TOFMS, the baseline noise comes from the shot noise of all ion 
species; thus, both PHP and SNR are equal, whereas in on-axis TOF, the SNR can become infinite in the absence of detector noise. Therefore, PHP is a better figure of merit for comparing HT-TOF to on-axis TOF. As shown in Ref. 5, this ratio normally exceeds unity and thus represents an enhancement except for ions that are a very small fraction (typically less than 0.001 ) of the total ion signal. We have also shown [5] that the peak height and the variance are given by

$$
\begin{aligned}
& \left\langle z_{i}\right\rangle \approx(1-\delta) F_{i} f_{\mathrm{HT}} \Psi_{1}^{\mathrm{S}} \\
& \operatorname{Var}\left(z_{i}\right) \approx f_{\mathrm{HT}} \Psi_{1}^{\mathrm{S}}
\end{aligned}
$$

where $\Psi_{1}^{S}$ is the total ion counts during the spectral acquisition time when the BNG is fixed in the transmission mode (sequence element 1 ), and $\delta$ is the proportion of unmodulated ions arising from incomplete deflection of the BNG.

It is important to note that the performance of HT-TOFMS depends on the duty cycle and the ion modulation efficiency $(1-\delta)$. Both are determined by the ion beam size, the detector dimensions, and the BNG parameters that control ion deflection. To study the effects of ion modulation on the performance, simple analytical equations, which accurately describe the ion deflection by the BNG, have been derived and compared with SimIon simulations and beam imaging experiments. These expressions can be used to model the dynamics of the ion beam deflection as the BNG is switched on and off. Ion currents in both channels of the 2C-HT-TOFMS, in response to BNG switching, can be calculated by integrating over the ion beam distribution and detector dimensions for various BNG parameters, ion beam characteristics, and detector dimensions. This study provides us with a deeper understanding of the ion modulation process and its effects on HT-TOFMS, and it also allows us to determine for a given set of parameters the optimum condition for HT-TOFMS.

\section{Experimental}

\section{Reagents}

All experiments were done with reserpine (Sigma Chemical, St. Louis, $\mathrm{MO}, \mathrm{Mw}=608.7 \mathrm{~g} \mathrm{~mol}^{-1}$ ). The reserpine concentrations were $1 \mathrm{mM}$ in a 50:50 vol/vol mixture of high-purity water (18 $\left.\mathrm{M} \Omega \mathrm{cm}^{-1}\right)$ and methyl alcohol (Aldrich Chemical, Milwaukee, WI). Analytes were used as received without further purification.

\section{Electrospray Ionization HT-TOFMS}

Figure 2a presents a schematic diagram of the HT-TOF mass spectrometer used in this work. A full description can be found elsewhere [5]. Analyte solutions were driven through 75-micron i.d. fused-silica capillaries (Polymicro Technologies, Phoenix, AZ) at flow rates between 0.1 and $1 \mu \mathrm{L} / \mathrm{min}$ by application of a backpres-

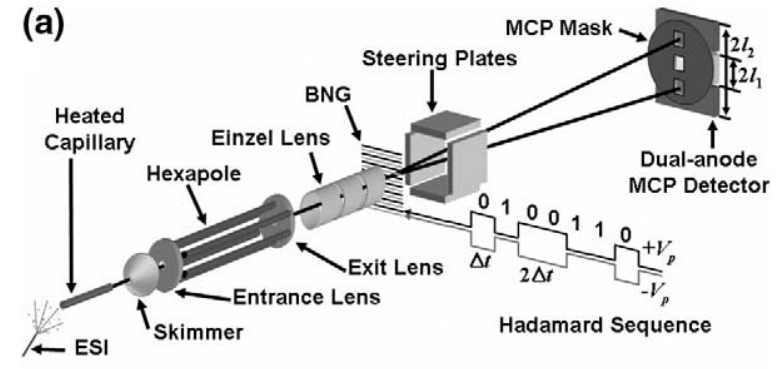

(b)
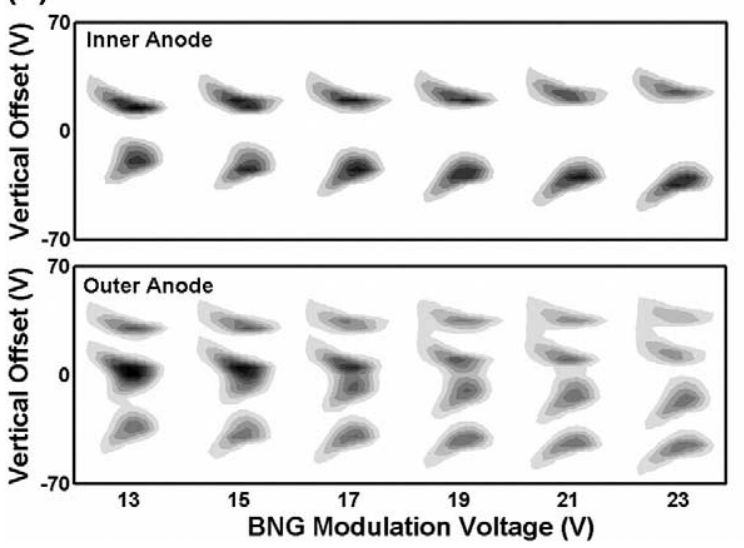

Figure 2. (a) Schematic of HT-TOFMS instrumentation. Electrosprayed ions are collimated by an rf-only hexapole ion guide $(1 \times$ $10^{-4}$ torr) and then accelerated to $1500 \mathrm{eV}$ between the exit lens of the hexapole and the first element of the Einzel lens. The Hadamard voltage sequence from the sequence generator is applied on the two wire sets of the BNG, which modulates the ion beam. The steering plates are used to scan the ion beam on the plane of the detector. The ions that are not blocked by the mask are detected by a dual-anode MCP detector. A multichannel scaler records the signals from both anodes. (b) Ion beam images at BNG voltages of $\pm 13,15,17,19,21$, and $23 \mathrm{~V}$. Beam images were collected by fixing the BNG voltage and recording the total ion counts while stepping the horizontal and vertical voltage offsets of the steering plates. The images at the inner anode clearly show the ion beam deflection by the BNG. The images at the outer anode show four branches caused by the two openings on the MCP mask.

sure and were electrosprayed from the exit, which was sharpened and gold-coated [9]. The electrospray tip was held at voltages between 2500 and $3500 \mathrm{~V}$ and positioned roughly $5 \mathrm{~mm}$ from the grounded entrance orifice. The electrosprayed ions are collimated in an rf-only hexapole (2.9 MHz, 200 to $2500 \mathrm{~V}$, ABB Inc, Pittsburgh, PA), which is maintained at a pressure of 1 $\times 10^{-4}$ torr, before being accelerated to $1500 \mathrm{eV}$ between the exit lens of the hexapole and the first element of the Einzel lens that follows the hexapole.

Encoding sequences are applied to the BradburyNielson gate, which is mounted just past the Einzel lens. The wires of the gate $(20-\mu \mathrm{m}$ diameter gold-plated tungsten, $100-\mu \mathrm{m}$ spacing) float at the acceleration voltage of the instrument and are aligned such that beam deflection is along the vertical axis. High voltage and deflection bias voltages are supplied to the gate by a HT-TOFMS control system that was engineered in partnership with Predicant Biosciences (South San 
Francisco, CA). This PC-controlled system consists of a central control box that generates variable-length, variable-rate pseudorandom sequences and vacuummounted driver circuitry that applies the acceleration voltage and the time-varying bias voltages to the BNG. For any applied encoding sequence, the temporal width of the encoding elements is the reciprocal of the sequence generator's clocking frequency. The total duration of one acquisition pass, which determines the mass range investigated, is the product of the element width and the sequence length. The time zero of an ion's flight time is defined as the moment it crosses the plane of the BNG wires.

The 25-mm dual-anode multichannel plate detector (MCP; Quantar Technology Inc., Santa Cruz, CA) was placed in a linear geometry. The total flight path past the BNG is $1.2 \mathrm{~m}$. Steering plates consisting of four plates $(30 \mathrm{~mm} \times 40 \mathrm{~mm})$ were used to position the ion beam at the detector. The inner anode of the MCP detector has a dimension of $4 \mathrm{~mm} \times 21 \mathrm{~mm}$ and the outer anode has a diameter of $25 \mathrm{~mm}$ with a rectangular hole of $5 \mathrm{~mm} \times 22 \mathrm{~mm}$. A mask having three openings ( $8 \mathrm{~mm}$ wide, $4 \mathrm{~mm}$ high) that are spaced vertically by 9 $\mathrm{mm}$ is mounted directly in front of the MCP detector for ion beam imaging experiments. The inner anode detects ions entering the central opening, whereas the outer anode detects the ions entering the outer openings. Other ions are blocked by the mask and do not reach the detector. Note that the purpose of the MCP mask is to study the ion modulation process. Under normal mode of operation, the mask should be removed. The MCP signals from the two anodes are amplified and fed into two multichannel scalers (Turbo MCS, EG and G Ortec, Oak Ridge, TN) for preparing histograms of the ion counts. Synchronization of the data acquisition with the modulation electronics is achieved by triggering the start pulse of the data acquisition from the sequence generator at the beginning of the first sequence element. The digitized waveform acquired by the multichannel scaler is transferred to a computer. All data processing is performed with a program written in Python programming language.

\section{Beam Imaging}

The ion counts at both MCP anodes were monitored while scanning the voltages applied on the steering plates to acquire the ion beam images in Figure $2 \mathrm{~b}$. Changing the voltage difference of the horizontal and vertical plates deflects the ion beam horizontally and vertically. The BNG voltage was fixed at $\pm 13 \mathrm{~V}$, and the two voltage offsets of the steering plates were increased in $5-\mathrm{V}$ steps after summing the total ion count for $5 \mathrm{~s}$ from -70 to $70 \mathrm{~V}$. The scanning was repeated for BNG voltages of 15, 17, 19, 21, and $23 \mathrm{~V}$. The ion beam images are a convolution of the ion beam shape and the MCP mask shape (three openings that are $8 \mathrm{~mm}$ wide and 4 $\mathrm{mm}$ high). The images from the inner anode show the two ion beam branches deflected by the BNG. The outer anode has two mask openings that are separated by 18 $\mathrm{mm}$. Thus, the images from the outer anode have four branches.

\section{Theory}

\section{Ion Trajectory on the BNG Potential Energy Surface}

An analytical expression for the potential energy surface of the BNG was first derived in 1929 by Bethe [10]. It also appears in the thesis of Yang [11], who used conformal mapping. It is possible to re-express their results as

$$
U(x, y)=\frac{k V_{\mathrm{p}}}{\pi} \ln \left[\frac{\cosh \left(\frac{\pi x}{d}\right)-\sin \left(\frac{\pi y}{d}\right)}{\cosh \left(\frac{\pi x}{d}\right)+\sin \left(\frac{\pi y}{d}\right)}\right]
$$

where $U(x, y)$ is the potential energy surface of the BNG, and the constant $k$ is defined as

$$
k \equiv \frac{\pi}{2 \ln \left[\cot \left(\frac{\pi R}{2 d}\right)\right]}
$$

Here, the BNG is on the $\mathrm{y}-\mathrm{z}$ plane (Figure 1c), and $d$ and $R$ are the distance between the wires and the radius of the wires, respectively. In eq 7, positive ions are deflected in a positive direction.

The trajectory of an ion can be calculated by solving the Newton's equation on the BNG potential energy surface. The ion is assumed to be traveling in the positive $\mathrm{x}$-axis direction.

$$
\ddot{x}=-\frac{z e}{M} U^{\prime} \quad \ddot{y}=-\frac{z e}{M} \frac{\partial U}{\partial y} .
$$

Here, a dot refers to a time derivative and a prime refers to differentiation with respect to $x$. Also, $z, e$, and $M$ are the number of charges on the ion, the charge of an electron, and the mass of the ion, respectively. From conservation of energy, the kinetic energy $z e V(x, y)$ is related to the BNG potential by

$$
z e V(x, y)=\frac{1}{2} M\left(\dot{x}^{2}+\dot{y}^{2}\right)=z e V_{0}-z e U(x, y)
$$

where $z e V_{0}$ is the kinetic energy when $\mathrm{U}(x, y)=0$. Using eq 10 and through change of variables in eq 9, a differential equation for the ion trajectory can be obtained:

$$
y^{\prime \prime}+\frac{1+y^{\prime 2}}{2 V}\left(V^{\prime} y^{\prime}-\frac{\partial V}{\partial y}\right)=0
$$

Note that the kinetic energy $V$ is used instead of $U$ to be 
more consistent with the paraxial equation for electron motion under cylindrical potential energy surface [12]. Eq 11 can be greatly simplified if the kinetic energy of the ion is much greater than the voltage applied on the BNG $\left(V_{0} \gg U\right)$. In this case, we can assume the following.

$$
V^{\prime} \approx 0 \quad y^{\prime 2} \ll 1
$$

The kinetic energy of the ion $z e V_{0}$ is $1500 \mathrm{eV}$, and the voltage applied on the BNG is around $13 \mathrm{~V}$, which is less than $1 \%$ of the ion kinetic energy. Thus, using eq 7 , eq 11 can be simplified to

$$
y^{\prime \prime}=\frac{1}{2 V_{0}} \frac{\partial V}{\partial y}=\frac{k V_{\mathrm{p}}}{d V_{0}} \frac{\cosh (\pi x / d) \cos (\pi y / d)}{\cosh ^{2}(\pi x / d)-\sin ^{2}(\pi y / d)}
$$

This equation expresses the deflection power of the BNG. The tangent of the deflection angle $\tan \alpha$ can be calculated by integrating this equation. Let us imagine that the voltages on the wires of the BNG are at $\pm V_{p}$, and that an ion with a kinetic energy $z e V_{0}$ approaches the BNG from $\left(-\infty, y_{0}\right)$. If the voltages of the BNG are suddenly turned on when the ion is at position $\left(x_{0}, y_{0}\right)$ and turned off at $\left(x_{1}, y_{0}\right)$, then, the deflection angle is

$$
\begin{aligned}
\tan \alpha\left(x_{0}, x_{1} ; y_{0}\right) & =\int_{x_{0}}^{x_{1}} y^{\prime \prime} d x \\
& =\frac{k V_{\mathrm{p}}}{d V_{0}} \int_{x_{0}}^{x_{1}} \frac{\cosh (\pi x / d) \cos (\pi y / d)}{\cosh ^{2}(\pi x / d)-\sin ^{2}\left(\pi y_{0} / d\right)} d x \\
& =k \frac{V_{\mathrm{p}}}{V_{0}}\left[\Theta\left(x_{1}, y_{0}\right)-\Theta\left(x_{0}, y_{0}\right)\right]
\end{aligned}
$$

where $\alpha\left(x_{0}, x_{1}, y_{0}\right)$ is the deflection angle and the normalized deflection $\Theta(x, y)$ is defined as

$$
\begin{aligned}
\Theta(x, y) \equiv & \frac{1}{\pi}\left[\arctan \left(\frac{e^{\pi x d}+\sin (\pi y / d)}{\cos (\pi y / d)}\right)\right. \\
& \left.+\arctan \left(\frac{e^{\pi x d}-\sin (\pi y / d)}{\cos (\pi y / d)}\right)\right]
\end{aligned}
$$

The maximum deflection is given by

$$
\tan \alpha_{\max }=\tan \alpha\left(-\infty, \infty ; y_{0}\right)=k \frac{V_{\mathrm{p}}}{V_{0}}
$$

where according to eq $8, k$ depends only on the ratio $R / d$. Eq 14 is a central result of our analysis. Later, we shall present an expression obtained by averaging over all possible values of $y_{0}$.

\section{Comparison with SimIon Simulations}

To check the accuracy of the analytical equations, ion trajectories through the BNG were simulated using SimIon 3D v7.0 [13]. In all the simulations, the BNG was
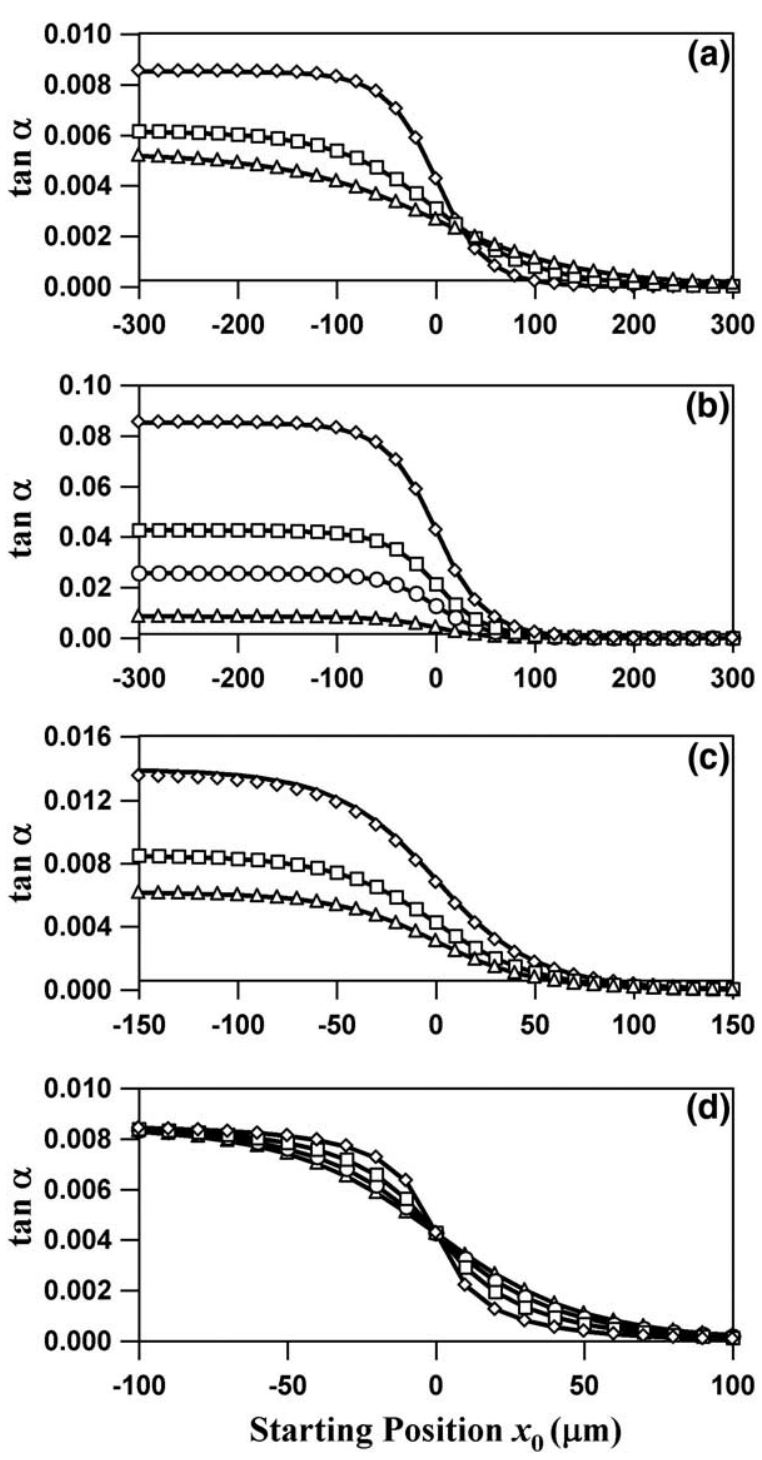

Figure 3. Plots of BNG deflection angle versus starting position. All markers are from SimIon simulations and lines are predictions from eq 14. (a) $\tan \alpha$ for wire spacings of $d=100$ (open diamond), 200 (open square), and $300 \mu \mathrm{m}$ (open triangle). Other parameters are $M=10 \mathrm{kDa}, V_{p}=10 \mathrm{~V}, V_{0}=1000 \mathrm{~V}$, and $R=10 \mu \mathrm{m}$. (b) tan $\alpha$ at deflection voltages of $V_{p}=10$ (open triangle), 30 (open circle), 50 (open square), and $100 \mathrm{~V}$ (open diamond). Other parameters are $M=10 \mathrm{kDa}, R=10 \mu \mathrm{m}, d=100 \mu \mathrm{m}$, and $V_{0}=1000 \mathrm{~V}$. (c) tan $\alpha$ for a wire radius $R$ of 5 (open triangle), 10 (open square), and 20 $\mu \mathrm{m}$ (open diamond). Other parameters are $M=10 \mathrm{kDa}, V_{p}=10$ $\mathrm{V}, V_{0}=1000 \mathrm{~V}$, and $d=100 \mu \mathrm{m}$. (d) $\tan \alpha$ at different initial $y$ position of $y_{0}=0$ (open triangle), 20 (open circle), 30 (open square), and $39 \mu \mathrm{m}$ (open diamond). Other parameters are $M=10$ $\mathrm{kDa}, R=10 \mu \mathrm{m}, d=100 \mu \mathrm{m}, V_{p}=10 \mathrm{~V}$, and $V_{0}=1000 \mathrm{~V}$.

along the $y$-axis (Figure 1c) and 50 ions were placed on the $\mathrm{x}$-axis spaced by 10 or $20 \mu \mathrm{m}$. The wire radius $R$ was 5,10 , or $20 \mu \mathrm{m}$, and the wire spacing $d$ was 100, 200, or $300 \mu \mathrm{m}$. The ions had a kinetic energy of $1000 \mathrm{eV}$ flying in the positive $x$-axis direction. The alternate wires had the voltages $V_{\mathrm{p}}$ of $\pm 10,30,50$, or $100 \mathrm{~V}$. Figure 3 presents the deflection angle at $\mathrm{x}=1000 \mathrm{~mm}$ plotted against the starting x-position. The starting position $x_{0}$ 
is the $x$-position of the ions relative to the BNG when the voltages are suddenly applied to the BNG wires.

$$
\tan \alpha\left(x_{0}, \infty ; y_{0}\right)=k \frac{V_{\mathrm{p}}}{V_{0}}\left[1-\Theta\left(x_{0}, y_{0}\right)\right]
$$

When the BNG is switched from on to off or from off to on, ions that are close to the BNG will be partially deflected leading to a blurring of the deflection, whereas those ions far away are unaffected. This behavior is shown in Figure 3a, where the deflection angle is plotted for three different wire spacings $d$. The markers refer to the SimIon simulations and the solid lines are from eq 17. Smaller spacing causes larger deflection angles and less blurring. The ions that are away from the BNG by more than the wire spacing are either deflected fully or not deflected. The ions that are within a distance of the wire spacing from the BNG are partially deflected. In Figure $3 b$ and $3 c$, the voltage $V_{\mathrm{p}}$ and the wire radius $R$ are varied, respectively and in Figure $3 d$, the initial y-position $y_{0}$ is varied from the center of the wire spacing. The wire spacing $d$ determines the extent of blurring, whereas the wire voltage $V_{\mathrm{p}}$ and the ratio of the wire radius $R$ to the wire spacing $d$ are responsible for the deflection angle. The deflection angle is fairly constant near the center of the wires and deviates only when $y_{0}$ is close to the wires. In all cases, the accuracy of the analytical equation is seen by the good fit with the SimIon simulations.

\section{Average Deflection Angle}

The ion beam is wider than the wire spacing of the BNG and will span several wires. Thus, it is more convenient to use the average of the deflection angles over all $y_{0}$ between the wires and remove the $y_{0}$ dependence. Figure 4 a shows the averages of deflection angles over $y_{0}$ for different starting $x$-positions. Each marker corresponds to the average of the SimIon simulation from 799 ions, which were placed at a fixed x-position with y ranging from -39.9 to $39.9 \mu \mathrm{m}$. This simulation was repeated for 13 different starting x-positions. On the right side of the plot is the histogram of the 799 ions. The gray bars are the SimIon simulations and the black lines are the predicted histograms from eq 14. These histograms display a very good fit between the simulations and the theory. To find the analytical expression for the average deflection angle, eq 14 has to be integrated over $y_{0}$. Instead, this equation was fit to a form almost identical to eq 14 with $y_{0}=0$ and the wire spacing $d$ replaced by an effective wire spacing $d_{\text {eff }}$.

$$
\begin{aligned}
& \tan \alpha_{\mathrm{av}}\left(x_{0}, x_{1}\right) \approx k \frac{V_{\mathrm{p}}}{V_{0}}\left[\Theta\left(x_{1}, 0 ; d_{\mathrm{eff}}\right)-\Theta\left(x_{0}, 0 ; d_{\mathrm{eff}}\right)\right] \\
& =k \frac{V_{\mathrm{P}}}{V_{0}}\left[\frac{2}{\pi} \arctan \left(e^{\pi x \gamma d_{\mathrm{eff}}}\right)-\frac{2}{\pi} \arctan \left(e^{\pi x 0 d d_{\mathrm{eff}}}\right)\right]
\end{aligned}
$$

The best fit is achieved when $d_{\text {eff }}$ is given by (a)
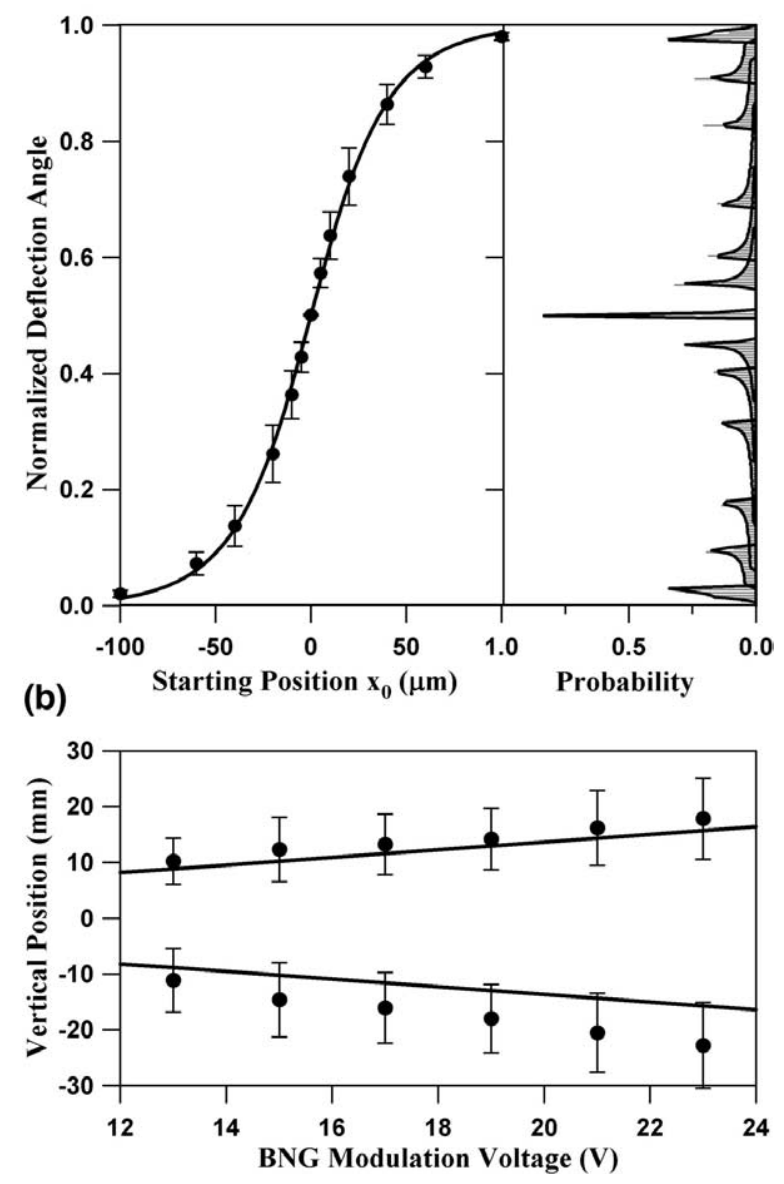

Figure 4. (a) Comparison of SimIon simulations and theory for the average deflection angle over $y_{0}$. Each marker on the left corresponds to the average deflection angle from a SimIon simulation of 799 ions starting at a position $\left(x_{0}, y_{0}\right)$, where $x_{0}$ is fixed and $y_{0}$ ranges from -39.9 to $39.9 \mu \mathrm{m}$. The solid curve on the left is calculated from eq 18. The histogram of SimIon's simulated deflection angles is shown on the right in gray. The predicted histogram calculated using eq 14 is shown as a black solid line on the right side of the plot. Some of the parameters were $M=10$ $\mathrm{kDa}, R=10 \mu \mathrm{m}, d=100 \mu \mathrm{m}, V_{p}=13 \mathrm{~V}$, and $V_{0}=1500 \mathrm{~V}$. (b) Comparison of experiment and theory for the maximum deflection angle. The black dots representing the vertical positions were calculated from the beam images in Figure $2 \mathrm{~b}$. The voltage difference of the outer two branches of the outer-anode image was assumed to be the sum of the BNG beam deflection and the distance between two MCP mask openings $(18 \mathrm{~mm})$. The two branches in the inner-anode image are due to BNG deflection only. Thus, the difference of the inner and outer images was used to convert voltage to physical distance on the plane of the detector. The error bars are from the widths of the ion beam branches. The solid straight lines are from eq 16.

$$
d_{\mathrm{eff}}=d \cos \left(\frac{\pi(d-2 R)}{4 d}\right)
$$

The solid line in Figure $4 \mathrm{a}$ is the prediction from eqs 18 and 19 and show a very good fit with the SimIon simulations. These equations can accurately describe the average dynamic behavior of ions as they are deflected by the BNG. This equation can be extended to 
other geometry ion modulation gates by changing appropriately $k$ and $d_{\text {eff }}$. We consider these two equations to be a major result of this analysis. In the following sections, eqs 18 and 19 are used for all discussions.

\section{Beam Imaging Experiments}

Although the SimIon simulations are sufficient to validate the analytical expressions for the deflection angle given by eqs 14 and 18, two-dimensional images of ion beam deflection by the BNG were collected to compare with theory. As explained in the Experimental section, the images were acquired by scanning the steering plates and detecting with a masked dual anode multichannel plate detector (Figure 2b). The images are convolutions of the ion beam shape and the shape of the mask openings. The inner anode shows the two branches of the ion beam deflected by the BNG, whereas the outer anode shows four branches coming from the two outer openings on the mask.

To convert the voltage offsets of the steering plates into physical distance at the plane of the detector, the distance between the mask openings $(18 \mathrm{~mm}$ ) was used. The voltage difference between the outer two branches in the outer anode image was assumed to a sum of the ion beam deflection and the mask opening distance. The voltage difference between the two branches in the inner anode is only from the BNG deflection. Thus, the difference between the inner and the outer should correspond to a distance of $18 \mathrm{~mm}$. All ion beam branches were summed along the horizontal voltage offsets, and the centroid and the width of the ion beam branches along the vertical voltage offsets were calculated by fitting to a Gaussian function. Figure $4 \mathrm{~b}$ shows the average positions of the deflected ion beams for several BNG voltages (black dots). The error bars are from the width of the Gaussian fits that are corrected for the width of the mask openings. The solid lines are from eq 18 and are within the experimental error. SimIon simulations of the steering plates show that the steering plates cause some distortion of the beam images caused by the coupling of the horizontal and vertical plates. These distortions are probably the main cause of the slight deviations.

\section{Results and Discussion}

\section{Two-Channel Detection}

Common figures of merit that describe the performance of TOFMS are the signal-to-noise ratio (SNR), sensitivity, and mass resolution. In the current implementation of 2C-HT-TOFMS, the mass resolution is determined by the time width of the data acquisition bin $\Delta t$ and is not affected significantly by the ion deflection. In contrast, the SNR and sensitivity are directly related to the duty cycle and the ion modulation efficiency, and these depend strongly on the ion modulation process. Eqs 18 and 19, derived in the Theory section, accurately de- scribe the average deflection angle of an ion under the assumption that the kinetic energy of the ion is larger than the applied voltage. Here, we describe the effects of ion deflection on two-channel detection when a single voltage pulse is applied. This treatment will be expanded to the Hadamard sequence in the next section.

If a voltage pulse with time duration $\Delta t$ is applied on the BNG, the deflection angle as a function of time $t(\mathrm{t}$ $\left.=x_{0} / v_{0}+\Delta t / 2\right)$ can be easily derived from eq 18

$$
\begin{aligned}
\tan \alpha_{\mathrm{av}}(t ; \Delta t)= & k \frac{V_{\mathrm{p}}}{V_{0}}\left[\frac{2}{\pi} \arctan \left(e^{\pi v_{0}(t+\Delta t 2) d_{\mathrm{eff}}}\right)\right. \\
& -\frac{2}{\pi} \arctan \left(e^{\left.\pi v_{0}(t-\Delta t 2) d_{\mathrm{eff}}\right)}\right] \\
= & k \frac{V_{\mathrm{p}}}{V_{0}} \frac{2}{\pi} \arctan \left[\sinh \left(\frac{\pi v_{0} \Delta t}{2 d_{\text {eff }}}\right) / \cosh \left(\frac{\pi v_{0} t}{d_{\text {eff }}}\right)\right]
\end{aligned}
$$

where $v_{0}=\sqrt{\frac{2 z e V_{0}}{M}}$ is the velocity of the ion. The time zero has been chosen to be the center of the pulse.

In 2C-HT-TOFMS, the ions are detected by a dualanode MCP detector positioned at $x=L$ (Figure 2a). The two-channel detection will be determined by the $y$-position of the ions at the plane of the detector $y_{\text {Det }}$ and it can be calculated from

$$
y_{\text {Det }}=y_{0} \pm L \tan \alpha(t ; \Delta t)
$$

where $L$ is the distance between the BNG and the detector and $y_{0}$ is the initial position in the plane of the BNG. Note that $y_{0}$ refers to the y-position within an ion beam distribution and ignores the microstructure of the BNG. During the sequence state 1 , the BNG wires are at relative ground, and $\tan \alpha=0$. During state 0 , the BNG deflects the ion beam into two branches; thus, two $y_{\text {Det }}$ values are given. If the sizes of the inner and outer anodes are $2 l_{1}$ and $2\left(l_{2}-l_{1}\right)$, respectively (Figure $2 a$ ), the inner channel will detect the ions that reach the inner anode $\left(\left|y_{\text {Det }}\right|<l_{1}\right)$ and the outer channel will detect those that arrive at the outer anodes $\left(l_{1}<\left|y_{\text {Det }}\right|<l_{2}\right)$. In 1C-HT-TOFMS, only the inner channel is used.

An ion beam has a finite size and $y_{0}$ will have a distribution. If the distribution is larger than the detector, the performance is degraded. Let us assume that the ion beam has a probability distribution of a hyperbolic secant

$$
f\left(y_{0}\right)=\frac{1}{\pi \Delta y} \operatorname{sech}\left(\frac{y_{0}-y_{m}}{\Delta y}\right)
$$

where $y_{\mathrm{m}}$ is the center and $2 \Delta y$ is the width at $e^{-1}$ of the maximum height. This distribution was chosen because it is analytically integrable and qualitatively similar to a 
Gaussian distribution. Thus, the requirements for the ion beam to strike either the inner channel or the outer channel detector are given by

$$
-l_{1} \mp L \tan \alpha_{\mathrm{av}}<y_{0}<l_{1} \mp L \tan \alpha_{\mathrm{av}}
$$

and

$$
\begin{gathered}
-l_{2} \mp L \tan \alpha_{\mathrm{av}}<y_{0}<-l_{1} \mp L \tan \alpha_{\mathrm{av}} \\
l_{1} \mp L \tan \alpha_{\mathrm{av}}<y_{0}<l_{2} \mp L \tan \alpha_{\mathrm{av}}
\end{gathered}
$$

respectively. The ion counts from the positive and negative branches at the inner channel are given by

$$
\begin{aligned}
I_{\mathrm{in}}^{ \pm}(t ; \Delta t)= & \frac{I_{0}}{2} \int_{-l_{1} \mp L \tan \alpha_{\mathrm{av}}(t ; \Delta t)}^{l_{1} \mp \tan \alpha_{\mathrm{av}}(t ; \Delta t)} \frac{1}{\pi \Delta y} \operatorname{sech}\left(\frac{\mathrm{y}_{0}-y_{\mathrm{m}}}{\Delta y}\right) d y_{0} \\
= & \frac{I_{0}}{2} \frac{2}{\pi}\left[\arctan \left(e^{\left(l_{1}-y_{\mathrm{m}} \mp L \tan \alpha_{\mathrm{av}}(t ; \Delta t)\right) \Delta y}\right)\right. \\
& \left.-\arctan \left(e^{\left(-l_{1}-y_{\mathrm{m}} \mp L \tan \alpha_{\mathrm{av}}(t ; \Delta t)\right) \Delta y}\right)\right] \\
= & \frac{I_{0}}{\pi} \arctan \left[\sinh \left(\frac{l_{1}}{\Delta y}\right) /\right. \\
& \left.\cosh \left(\frac{y_{\mathrm{m}} \mp L \tan \alpha_{\mathrm{av}}(t ; \Delta t)}{\Delta y}\right)\right]
\end{aligned}
$$

where $I_{0}$ is the maximum number of ion counts. The total ion counts at the inner channel is the sum of both branches

$$
I_{\text {in }}(t ; \Delta t)=I_{\text {in }}^{+}(t ; \Delta t)+I_{\text {in }}^{-}(t ; \Delta t)
$$

The ion counts at the outer channel can be calculated by replacing $l_{1}$ with $l_{2}$ and then subtracting the ion counts at the inner channel.

$$
\begin{aligned}
& I_{\mathrm{out}}^{ \pm}(t ; \Delta t) \\
& =\frac{I_{0}}{\pi} \arctan \left[\sinh \left(\frac{l_{2}}{\Delta y}\right) / \cosh \left(\frac{y_{m} \mp L \tan \alpha_{\mathrm{av}}(t ; \Delta t)}{\Delta y}\right)\right] \\
& \quad-I_{\mathrm{in}}^{ \pm}(t ; \Delta t)
\end{aligned}
$$

The total ion counts at the outer detector is

$$
I_{\text {out }}(t ; \Delta t)=I_{\text {out }}^{+}(t ; \Delta t)+I_{\text {out }}^{-}(t ; \Delta t)
$$

\section{Duty Cycle and Ion Modulation Efficiency}

The Hadamard sequence consists of multiple voltage pulses of various lengths and the dynamic behavior at every rising and falling edge affects the performance of HT-TOFMS. For a sequence of length $N=2^{n}-1$, it contains $(N+1) / 21$ s and $(N-1) / 2$ ss. The BNG wires are at relative ground for the sequence element 1 , and the wires are at voltages of $\pm V_{p}$ for the sequence element 0 . If the time width of the modulation element is $\Delta t$, there are $2^{n-3}$ voltage pulses of length, $\Delta t, 2^{n-4}$ pulses of length $2 \Delta t$, etc.

HT-TOFMS is an ion counting technique, which uses a voltage-discriminated multichannel scaler (MCS) to digitize the output of the MCP detector. The data acquisition bin time is usually chosen to be the same as the minimum voltage pulse width from the sequence generator. Ideally, the ion counts for the sequence element 0 should be low, but the modulation edges and large beam size cause blurring of the ion counts. The ion counts in each bin for a single voltage pulse of length $\Delta t$ can be calculated by integrating $I_{\text {in }}(t ; \Delta t)$ and $I_{\text {out }}(t ; \Delta t)$ from eqs 26 and 28 . The sequence element 1 corresponds to $\frac{\Delta t}{2}<|t|<\Delta t$ and the element 0 corresponds to $|t|<\frac{\Delta t}{2}$. Thus, the number of ion counts in the bin for elements 1 and 0 for a pulse of duration $\Delta t$ are

$$
\begin{aligned}
& \psi_{1}^{\Delta t}=\int_{-\Delta t}^{-\Delta t 2} I(t ; \Delta t) d t+\int_{\Delta t 2}^{\Delta t} I(t ; \Delta t) d t=2 \int_{\Delta t 2}^{\Delta t} I(t ; \Delta t) d t \\
& \psi_{0}^{\Delta t}=\int_{-\Delta t 2}^{\Delta t 2} I(t ; \Delta t) d t=2 \int_{0}^{\Delta t 2} I(t ; \Delta t) d t
\end{aligned}
$$

These are defined for both $I_{\text {in }}(t ; \Delta t)$ and $I_{\text {out }}(t ; \Delta t)$.

Let us define $\Psi_{1}$ and $\Psi_{0}$ as the total ion counts from all bins corresponding to sequence elements $1 \mathrm{~s}$ and $0 \mathrm{~s}$, respectively, when a Hadamard sequence is applied on the BNG wires. They are the sums of all ion counts from different length pulses.

$$
\begin{aligned}
\Psi_{1} & =\left(2^{n-3} \Psi_{1}^{\Delta t}+2^{n-4} \Psi_{1}^{2 \Delta t}+\cdots+\Psi_{1}^{(n-2) \Delta t}\right)+\Psi_{1}^{n \Delta t} \\
& =\Psi_{1}^{n \Delta t}+\sum_{k=1}^{n-2} 2^{n-2-k} \Psi_{1}^{k \Delta t} \\
\Psi_{0} & =\left(2^{n-3} \Psi_{0}^{\Delta t}+2^{n-4} \Psi_{0}^{2 \Delta t}+\cdots+\Psi_{0}^{(n-2) \Delta t}\right)+\Psi_{0}^{(n-1) \Delta t} \\
& =\Psi_{0}^{(n-1) \Delta t}+\sum_{k=1}^{n-2} 2^{n-2-k} \Psi_{0}^{k \Delta t}
\end{aligned}
$$

The sum $\Psi_{1}+\Psi_{0}$ is the total ion counts of a mass spectrum. We also need to define $\Psi_{1 \text {,static }}$ and $\Psi_{0 \text {,static }}$ which are the total ion counts during the acquisition time $(N \Delta t)$ when the state of the BNG is fixed in the states 1 or 0 , respectively. The values of $\Psi_{1 \text {,static }}$ and $\Psi_{0 \text {,static }}$ can be measured experimentally by fixing the BNG voltage and recording the ion counts. They can also be calculated analytically from $I_{\text {in }}(t)$ and $I_{\text {out }}(t)$ by replacing $\tan \alpha_{\text {av }}(t ; \Delta t)$ with 0 or $\tan \alpha_{\max }$ for the states 1 and 0 , respectively.

The duty cycle is defined as the ratio between the detected ion counts $\left(\Psi_{1}+\Psi_{0}\right)$ and the total ion counts that enter the mass analyzer $\left(\Psi_{\text {Tot }}\right)$. Thus, the duty cycles for the inner and outer channels are 


$$
f_{\mathrm{HT}}=\frac{\Psi_{1}+\Psi_{0}}{\Psi_{\mathrm{Tot}}}
$$

Note that the sample utilization efficiency is lower than the duty cycle by the transmission of the BNG grid, which is typically 80 to $90 \%$ (if $R=10 \mu \mathrm{m}$ and $d=100$ $\mu \mathrm{m}$, the transmission is $80 \%$ ). The duty cycle can be factored into a product of two terms.

$$
f_{\mathrm{HT}}=\frac{\Psi_{1}+\Psi_{0}}{\Psi_{\text {Tot }}}=\left(\frac{\Psi_{1}+\Psi_{0}}{\Psi_{1, \text { Static }}}\right)\left(\frac{\Psi_{1, \text { tatic }}}{\Psi_{\text {Tot }}}\right)
$$

The second term represents the ion beam clipping, which should equal 1 if the ion beam size is smaller than the detector. If the ion beam is larger than the detector, the tails of the ion beam are clipped off the detector and are not detected. The first term is the actual duty cycle of the modulation. Because it is easier to measure $\Psi_{1 \text {,static }}$ and the ion beam is smaller than the detector size under optimal conditions, the first term usually can be used as an approximation to the true duty cycle. In an ideal situation where $\Psi_{0}$ is negligible and there is minimal blurring of deflection angle at the voltage pulse edges, $\Psi_{1}+\Psi_{0} \approx[(N+1) / 2 N] \Psi_{1, \text { static }} \approx$ $(50 \%) \Psi_{1, \text { static }}$ and therefore, $f_{\mathrm{HT}}$ is ideally $50 \%$ for the one-channel experiment.

The modulation efficiency is defined as the percentage of ions that are actually modulated and contribute to the peak intensities. The ion counts that are in the state 0 only increase the baseline noise. The modulation efficiencies for the inner and outer channels are, thus, defined as

$$
\varepsilon=\frac{\left|\Psi_{1}-\Psi_{0}\right|}{\Psi_{1}+\Psi_{0}}
$$

The absolute value of the difference should be used because the outer channel detects the deflected branches of the ion beam, and the states 1 and 0 have opposite roles. In the limit of minimal blurring at the edges, the modulation efficiency will approach the static modulation efficiency defined as

$$
\varepsilon_{\text {Static }}=\frac{\left|\Psi_{1, \text { Static }}-\Psi_{0, \text { Static }}\right|}{\Psi_{1, \text { Static }}+\Psi_{0, \text { Static }}}
$$

Because it is easy to measure $\Psi_{1, \text { Static }}$ and $\Psi_{0, \text { Static }}$ experimentally, the static modulation efficiency $\varepsilon_{\text {State }}$ is sometimes used as an approximation to the dynamic modulation efficiency $\varepsilon$. However, $\varepsilon_{\text {Static }}$ only considers the effects from large beam size and do not take into account the dynamic behavior of the deflection.

In 2C-HT-TOFMS, the dominant source of noise is the shot noise and it is evenly spread throughout the mass spectrum during the deconvolution [5]. As shown in eq 6, the variance of the ion counts in the $i^{\text {th }}$ acquisition bin for each channel is given by

$$
\operatorname{Var}\left(z_{i}\right)=\Psi_{1}+\Psi_{0}=\frac{\Psi_{1}+\Psi_{0}}{\Psi_{\text {Tot }}} \Psi_{\text {Tot }}=f_{\mathrm{HT}} \Psi_{\text {Tot }}
$$

The variance is independent of the relative ion abundance and is the total ion counts in the mass spectrum. Therefore, the noise level is determined by the duty cycle $f_{\mathrm{HT}}$.

From eq 5 , the peak heights of the $i^{\text {th }}$ acquisition bin in the inner and outer detection channels are given by

$$
\begin{aligned}
\left\langle z_{i}\right\rangle & =F_{i}\left|\Psi_{1}-\Psi_{0}\right| \\
& =F_{i} \frac{\left|\Psi_{1}-\Psi_{0}\right|}{\Psi_{1}+\Psi_{0}} \frac{\Psi_{1}+\Psi_{0}}{\Psi_{\text {Tot }}} \Psi_{\text {Tot }} \\
& =F_{i} \varepsilon \varepsilon_{\mathrm{HT}} \Psi_{\text {Tot }}
\end{aligned}
$$

where $F_{i}$ is the relative abundance of the $i^{\text {th }}$ ion species [5]. The peak height is, therefore, determined by the relative abundance $F_{i}$, the modulation efficiency $\varepsilon$, and the duty cycle $f_{\mathrm{HT}}$.

The SNR is the ratio of the peak intensity to the baseline noise. A better figure of merit for HT-TOFMS in the shot-noise limit is the peak height precision (PHP) defined as the ratio between the peak intensity and its standard deviation [5]. Thus, the PHPs for both channels are

$$
\mathrm{PHP}_{i}=\frac{\left\langle z_{i}\right\rangle}{\sqrt{\operatorname{Var}\left(z_{i}\right)}}=\frac{F_{i} \varepsilon f_{\mathrm{HT}} \Psi_{\mathrm{Tot}}}{\sqrt{f_{\mathrm{HT}} \Psi_{\mathrm{Tot}}}}=F_{i} \varepsilon \sqrt{f_{\mathrm{HT}} \Psi_{\mathrm{Tot}}}
$$

The duty cycle $f_{\mathrm{HT}}$ appears in the expressions for the peak height, the baseline noise, and the peak height precision. Thus, higher duty cycle leads to increased peak height and also to larger baseline noise. The duty cycle can be expressed as a sum of two components

$$
f_{\mathrm{HT}}=\varepsilon f_{\mathrm{HT}}+(1-\varepsilon) f_{\mathrm{HT}}
$$

The first component appears in eq 38 and contributes to both the peak height and the baseline noise, whereas the second component only increases the baseline noise. Thus, a high duty cycle must be accompanied by a high modulation efficiency to capture the multiplexing advantage. Therefore, the best experimental conditions for HT-TOFMS are those that yield both high modulation efficiency and high duty cycle.

\section{Understanding and Optimizing 2C-HT-TOFMS}

The ion counts at both channels calculated using eqs 26 and 28 illustrate qualitatively the effects of edge blurring caused by various experimental parameters. Thus, the analytical expressions are very powerful tools for understanding the ion modulation process in 2C-HTTOFMS and for finding optimum experimental conditions.

Using eqs 26 and 28 , the duty cycles and modulation efficiencies were calculated at various conditions re- 
(a)
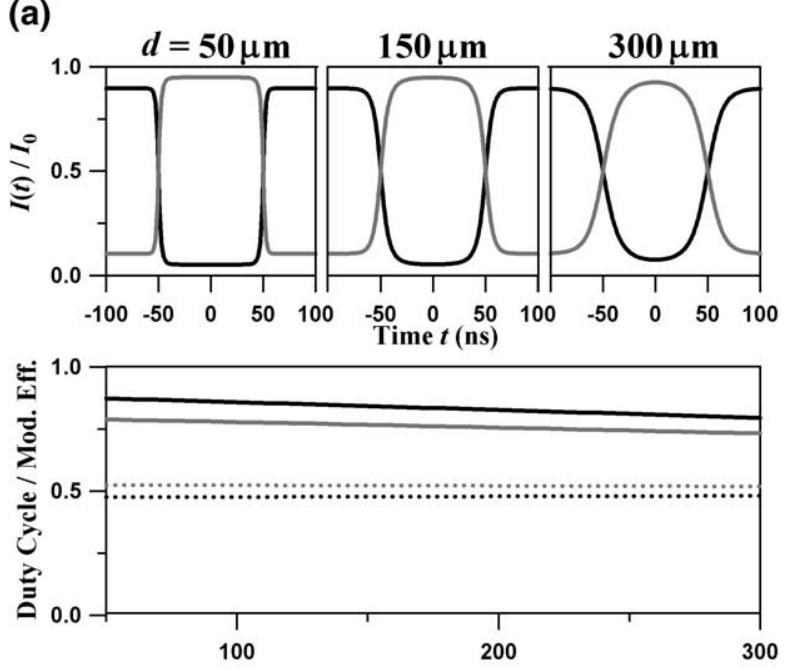

(b)
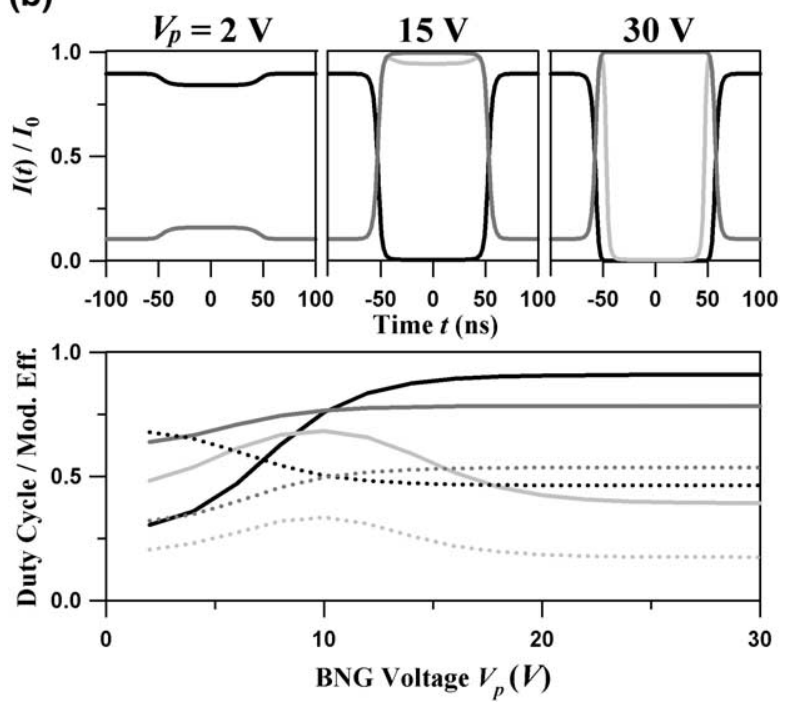

Figure 5. The effect of BNG parameters on the ion counts $I(t)$, the duty cycle, and the modulation efficiency. Black and gray lines are for the inner and outer channels, respectively. Other parameters are $\tan \alpha_{\max }=2 l_{1}, \Delta y=0.4 l_{1}, M=10,000 \mathrm{u}$, and $V_{0}=1500 \mathrm{eV}$. (a) The ion counts $I(t)$, duty cycle (dotted lines), and modulation efficiency (solid lines) versus BNG wire spacing $d$. The black and gray lines correspond to inner and outer detector, respectively. $R / d$ was fixed at 0.1 for constant $\tan \alpha_{\max }$ and $V_{\mathrm{p}}=13 \mathrm{~V}$. (b) Similar to Figure $5 \mathrm{a}$ except that the variable is the BNG wire voltage $V_{\mathrm{p}}$. The dark and light gray lines are for two different outer detector sizes of $l_{2}=5 l_{1}$ and $l_{2}=2 l_{1}$, respectively.

lated to the BNG (Figure 5), the ion characteristics (Figure 6), the ion beam size (Figure 7), and the detector dimensions (Figure 8). In Figures 5 through 8, the black and gray lines correspond to the inner and outer channels, respectively. Normalized ion counts of both channels in response to a 100-ns voltage pulse are displayed at the top of all figures under various experimental conditions. Time period between -50 and $50 \mathrm{~ns}$ corresponds to the sequence element 0 and the other time period corresponds to the element 1 . Nonzero baselines arise from the large ion beam size chosen for the calculations, and blurred edges are derived from the ion modulation processes. Below the ion count plots, modulation efficiencies and duty cycles are displayed. The solid lines and the dotted lines are the modulation efficiencies and duty cycles, respectively.

Figure 5 shows the dependence of the ion counts, duty cycle, and modulation efficiency on the BNG parameters. First parameter is the BNG wire spacing $d$ (Figure 5a). The ratio of the wire radius $R$ and the wire spacing $d$ is fixed so that the maximum deflection $\tan \alpha_{\max }$ is constant. At small $d, I(t)$ is close to a square pulse, but as $d$ is increased, blurring of the rising and falling edges become more significant. The duty cycles for both channels are constant because edge blurring simply shifts ion counts from sequence element 1 to
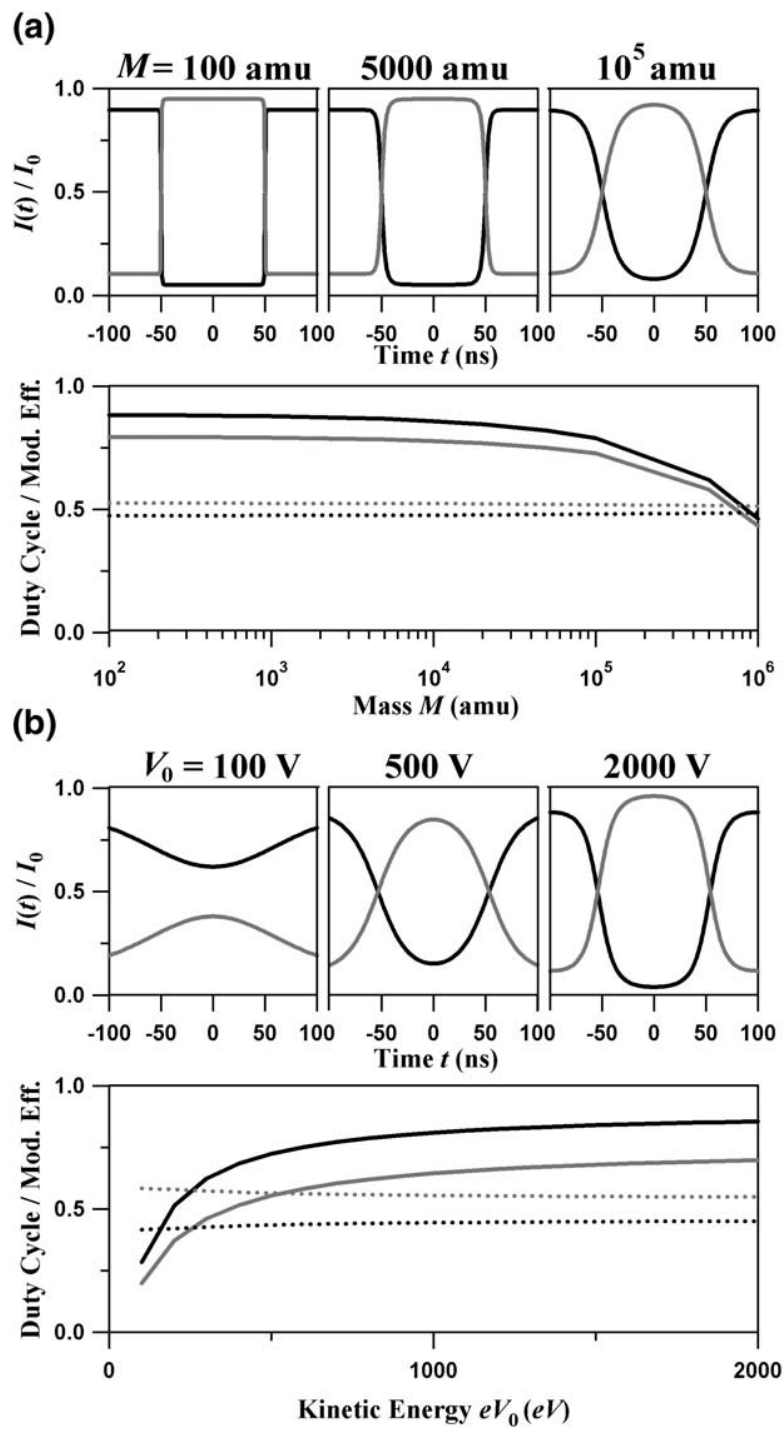

Figure 6. The effect of ion properties on the ion counts $I(t)$, the duty cycle (dotted lines), and the modulation efficiency (solid lines). Black and gray lines are for the inner and outer channels, respectively. Other parameters are $\tan \alpha_{\max }=2 l_{1}, \Delta y=0.4 l_{1}, d=$ $100 \mu \mathrm{m}, R=10 \mu \mathrm{m}$, and $V_{\mathrm{p}}=13 \mathrm{~V}$. (a) Mass dependence when $\mathrm{e} V_{0}=1500 \mathrm{eV}$. (b) Dependence on the kinetic energy of the ion $\mathrm{e} V_{0}$ when $M=200 \mathrm{kDa}$. 
(a)
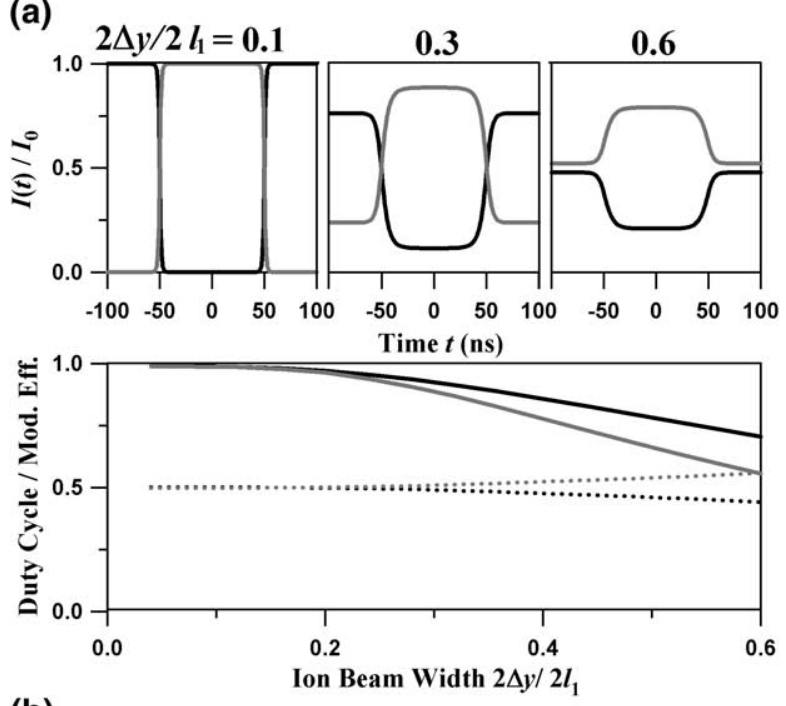

(b)
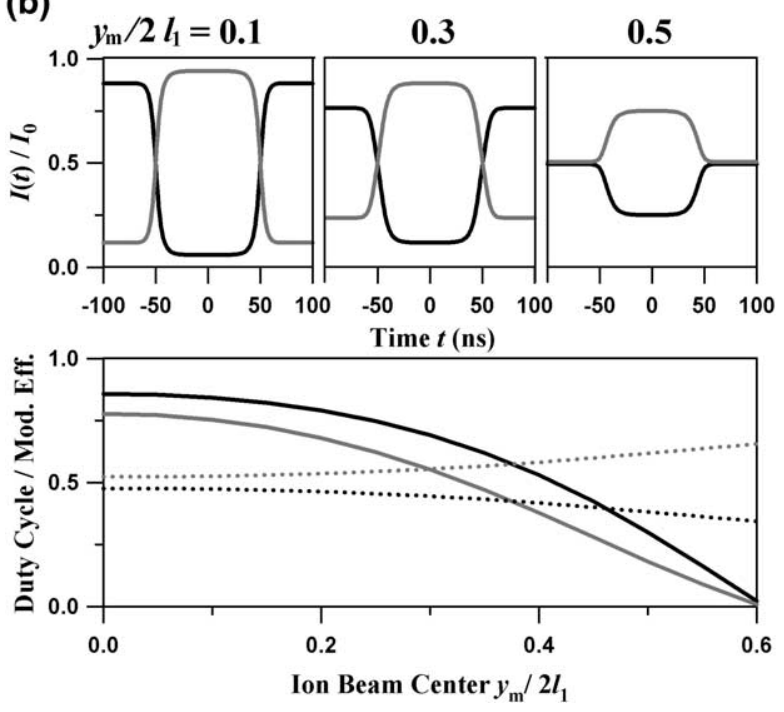

Figure 7. The effect of ion beam size on the ion counts $I(t)$, the duty cycle (dotted lines), and the modulation efficiency (solid lines). Black and gray lines are for the inner and outer channels respectively. Other parameters are $M=10,000 \mathrm{u}, d=100 \mu \mathrm{m}, R=$ $10 \mu \mathrm{m}, V_{\mathrm{p}}=13 \mathrm{~V}, V_{0}=1500 \mathrm{~V}, \tan \alpha_{\max }=2 l_{1}$, and $l_{2}=5 l_{1}$. (a) Dependence on the ion beam size $2 \Delta y$. (b) Dependence on the position of the center of the ion beam $y_{m}$.

element 0 , but this blurring reduces the modulation efficiencies as the wire spacing is increased. The second parameter is the BNG wire voltage $V_{\mathrm{p}}$ (Figure $5 \mathrm{~b}$ ). Here, dark and light gray lines are for large and small outer channel sizes, respectively. At very low $V_{\mathrm{p}}$, the ions are not sufficiently deflected and the ion beam stays in the inner channel. Thus, the duty cycle is very high for the inner channel, but the modulation efficiency is very low. The opposite is observed for the outer channel with high modulation efficiency and low duty cycle. As $V_{\mathrm{p}}$ is increased, the ion counts in sequence element 0 $\left(\Psi_{0}\right)$ decreases and the efficiency reaches the maximum. The duty cycles of both channels approach $50 \%$. When $V_{\mathrm{p}}$ is above $20 \mathrm{~V}$, ion deflection is excessive for a small outer channel such that the ion beam is deflected past the outer detector, causing the efficiency and the duty cycle to decrease. This sweeping appears as two separated peaks in $I(t)$. When combined with data acquisition oversampling, this mode of operation, known as differential impulse sweep mode, can be used to increase the mass resolution by sacrificing sensitivity [14]. Thus, in normal mode of operation, $V_{\mathrm{p}}$ must be above a threshold voltage, but too much voltage causes reduction in the modulation efficiency for the outer channel.

The ion properties such as mass and kinetic energy also influence the ion modulation. Figure 6a shows the mass dependence. As the mass of the ion species increases, the ions travel at a lower velocity spending more time passing the BNG. The edge blurring becomes more pronounced, and the modulation efficiency is

(a)
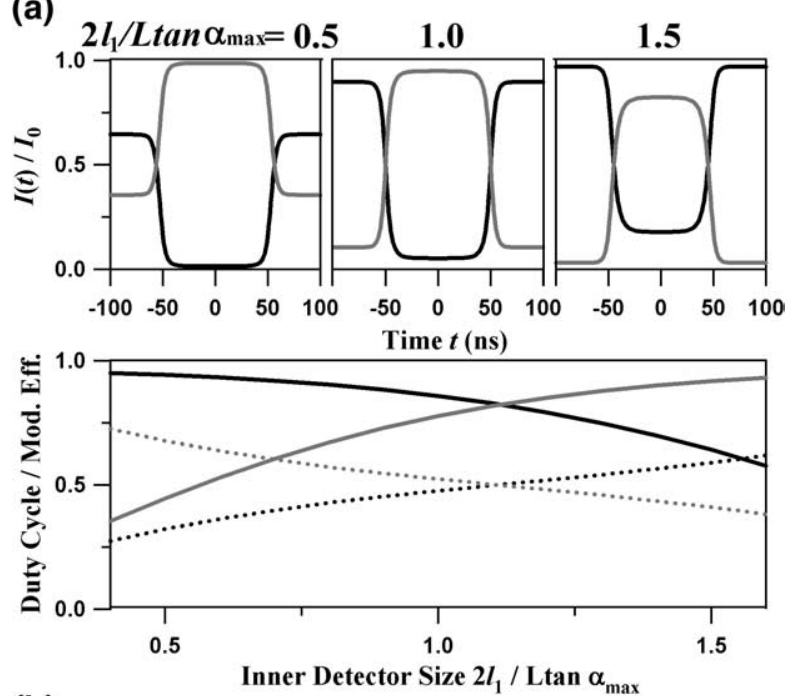

(b)
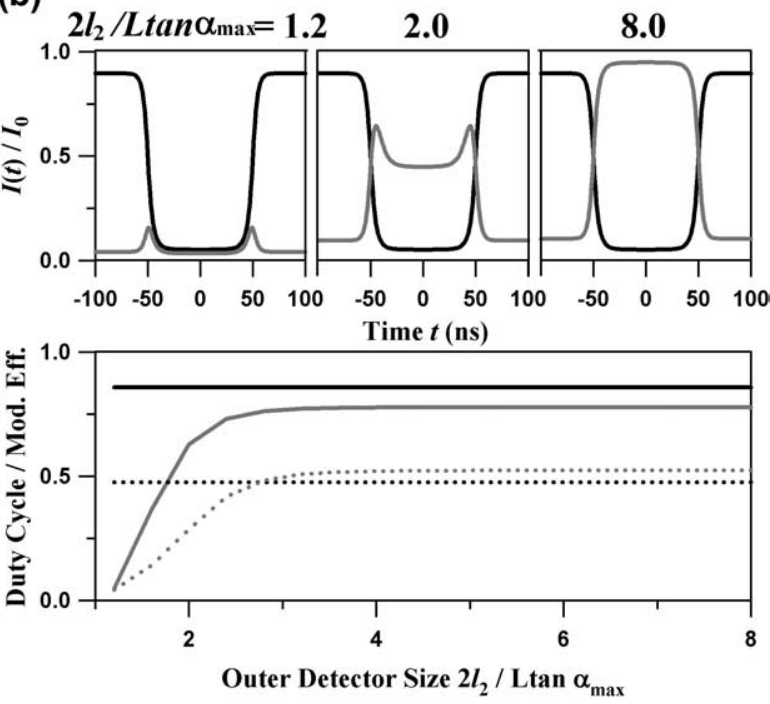

Figure 8. The effect of the detector dimensions on the ion counts $I(t)$, the duty cycle (dotted lines), and the modulation efficiency (solid lines). Black and gray lines are for the inner and outer channels respectively. Other parameters are $2 \Delta y=0.4 L \tan \alpha_{\max }$ $y_{m}=0, d=10 \mu \mathrm{m}, R=10 \mu \mathrm{m}, V_{\mathrm{p}}=13 \mathrm{~V}$, and $V_{0}=1500 \mathrm{~V}$. (a) Dependence on the inner channel detector size $2 l_{1}$. (b) Dependence on the outer channel detector size $2 l_{2}$. 
reduced for both channels. The duty cycles are constant because the ion beam size is assumed to be equal and the ion counts are simply shifted from element 1 to 0 . Thus, there is a mass cutoff, above which the modulation efficiency is significantly reduced. This mass cutoff has been reported previously [14], and sets an upper limit on the mass that can be analyzed using HTTOFMS. The mass cutoff $M_{\text {cutoff }}$ is given by the expression [15]

$$
M_{\text {cutoff }} \leq \frac{e V_{0} \Delta t^{2}}{2 d_{\text {eff }}^{2}}
$$

Under typical experimental conditions $\left(e V_{0}=1500 \mathrm{eV}\right.$, $d=100 \mu \mathrm{m}, \Delta t=100 \mathrm{~ns}$ ), theoretical $M_{\text {cutoff }}$ is $100 \mathrm{kDa}$. One method of reducing the mass dependence is to increase the kinetic energy $e V_{0}$. This dependence is plotted in Figure $6 \mathrm{~b}$ for $M=200 \mathrm{kDa}$. Here, the maximum deflection was fixed by keeping the ratio of $V_{\mathrm{p}}$ to $V_{0}$ constant. As $V_{0}$ is increased, the modulation efficiency approaches the maximum value.

Figure $7 \mathrm{a}$ shows the effects of the ion beam size on the modulation. The ion counts are almost perfect square pulses for very small ion beam sizes $(2 \Delta y)$. As the beam size is increased, the baseline increases and the edges are blurred. When the beam size is larger than half the size of the inner detector $\left(0.5 \times 2 l_{1}\right)$, the transmitted (state 1 ) and the fully deflected (state 0 ) ion beam begin to overlap and the modulation efficiency decreases appreciably. Thus, the ion beam deflection should be larger than twice the beam size. The ion beam center relative to the position of the detector is also very important as shown in Figure $7 \mathrm{~b}$. There is a sharp drop in the modulation efficiency when $y_{\mathrm{m}}$ is above $0.3 \times 2 l_{1}$. Therefore, the center of the ion beam should coincide with the center of the inner detector.

The detector dimensions are critical in 2C-HTTOFMS. Figure 8a shows the dependence of the inner channel detector size $\left(2 l_{1}\right)$. At small $2 l_{1}$, the modulation efficiency of the inner channel is high whereas that of the outer channel is low. This is because the ion counts of the inner channel in the sequence state 0 are almost zero, whereas the ion counts of the outer channel in the sequence state 1 are very large. However, the duty cycle is very low for the inner channel and very high for the outer channel as only a small fraction of the ion beam is sampled by the inner channel. The opposite behavior occurs at large $2 l_{1}$ where the modulation efficiency is high for the outer channel. The optimum is when the detector size is slightly larger than the maximum deflection. For 1C-HT-TOFMS, only the inner channel is used and, thus, smaller detector is preferred. In Figures $8 \mathrm{~b}$, the variable is the outer channel detector size $\left(2 l_{2}\right)$, which does not affect the ion counts in the inner channel. If the outer detector is too small, the ion beam is swept past the detector, and both the duty cycle and the modulation efficiency are low. The sum of duty cycles reaches the maximum of $100 \%$ when the outer channel detector is at least three times larger than the maximum deflection to detect all deflected ions.

These analyses demonstrate the importance of matching the dimensions of the detector size, ion beam size, and the ion beam deflection angle. The BNG voltage should be chosen such that the maximum deflection by the BNG is larger than the size of the inner detector and smaller than half the size of the outer detector such that all ions are detected. If the outer detector is large enough to detect all deflected ions, $100 \%$ duty cycle is always achieved. But, the modulation efficiency needs to be maximized by reducing the inner-anode ion counts in the sequence element 0 and the outer-anode ion counts in the element 1 . Tightly focusing the ion beam to be less than half the size of the inner detector and parking the beam in the center of the inner detector help achieve conditions of highest modulation efficiency. Smaller wire spacing for the BNG can also improve the modulation efficiency.

The main purpose of the analytical expressions for the ion modulation process is the qualitative understanding of 2C-HT-TOFMS. Many experimental factors were not considered in this paper such as kinetic energy and angular distribution, instrumental misalignment, and mechanical imperfections. Some of these distributions can be included by using larger ion beam size. By assuming different deflection voltages for the two ion beam branches deflected by the BNG, the expressions detailed in this paper can be used to approximate BNG or detector misalignment. This is demonstrated in Figure 9, where these analytical expressions were used to calculate the peak heights and baseline noises from experimental mass spectra from both channels, which were acquired as the vertical offset of the steering plates was scanned. Figure 9a shows the experimental mass spectra of reserpine in both channels. When the vertical offset is close to zero, the inner channel has the highest intensity. As the ion beam is moved off the center of the detector by the steering plates, the ion counts in sequence element 0 become larger than those in element 1, thus the peak height becomes negative. This indicates a negative modulation or a lack of modulated ion species relative to the high ion counts in state 0 . For the outer channel, the roles of state 1 and 0 are reversed such that the intensities are opposite those of the inner detector. Instrumental misalignment is evident from the different peak heights at vertical offsets of $\pm 20 \mathrm{~V}$. In Figure $9 \mathrm{~b}$, the absolute values of the peak heights and the variances of the baseline noise are plotted as black and gray dots for the inner and outer channels, respectively. The theoretical peak heights and variances were calculated from eq 37 and 38 using experimental parameters. The BNG voltages for the two ion beam branches were assumed to be different, and the two voltages (10 and $24 \mathrm{~V})$ and the total ion counts $\left(\Psi_{\text {Tor }}\right.$ $=1.1 \times 10^{6}$ ) were varied to give the best fit. The theoretical peak heights and variances for the inner 
(a)

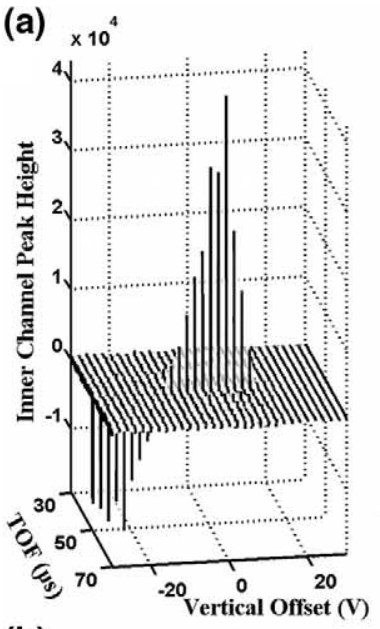

(b)

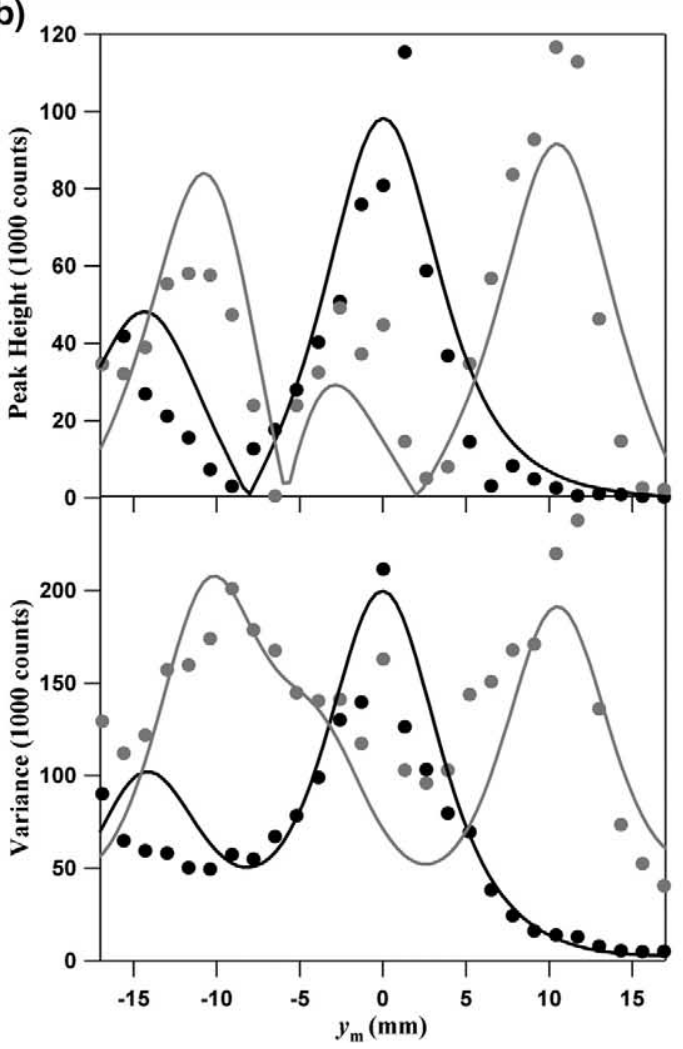

Figure 9. Mass spectra of reserpine versus the vertical offset of the steering plates. (a) Two-channel mass spectra of reserpine $(\mathrm{m} / \mathrm{z}$ $=609$ ). They were collected with 1023-element sequences, 100-ns acquisition bins $(10 \mathrm{MHz})$, and summed for $10 \mathrm{~s}$ per spectrum. The vertical offset voltage of the steering plates was changed from -30 $\mathrm{V}$ to $30 \mathrm{~V}$ in steps of $2 \mathrm{~V}$. The MCP mask had three openings $(8 \mathrm{~mm}$ wide and $4 \mathrm{~mm}$ high) that are spaced by $9 \mathrm{~mm}$. (b) The experimental peak area and the variance of the baseline noise are plotted for the inner (black dots) and the outer (gray dots) channels. Eqs 33 and 35 were used to calculate the duty cycles and modulation efficiencies. These were used to predict the peak heights and the variances from eqs 37 and 38 . The total ion counts $\left(\Psi_{\text {Tot }}\right)$ and the BNG voltages of the two deflected branches were used as parameters to fit the theory with experiments. The parameters were $2 \Delta y$ $=5.6 \mathrm{~mm}, \Psi_{\text {Tot }}=1.1 \times 10^{6}, V_{p}^{+}=10 \mathrm{~V}$, and $V_{p}^{-}=24 \mathrm{~V}$. and outer channels are shown as black and gray solid lines, respectively. The theoretical expressions agree qualitatively with the experiments and show the necessary features including the positive and negative peak heights, and misaligned side peaks.

\section{Comparison of One-Channel to Two-Channel HT-TOFMS}

In 2C-HT-TOFMS, the final mass spectrum is the sum of the spectra from both channels. The peak height and the variance of the noise for two-channel mass spectra are given by

$$
\begin{aligned}
& \left\langle z_{i}^{2 \mathrm{C}}\right\rangle=F_{i}\left(\varepsilon^{\text {in }} f_{\mathrm{HT}}^{\text {in }}+\varepsilon^{\text {out }} f_{\mathrm{HT}}^{\text {out }}\right) \Psi_{\text {Tot }} \\
& \operatorname{Var}\left(\mathrm{z}_{i}^{2 \mathrm{C}}\right)=\left(f_{\mathrm{HT}}^{\text {in }}+f_{\mathrm{HT}}^{\text {out }}\right) \Psi_{\text {Tot }}
\end{aligned}
$$

Thus, the PHP is given by

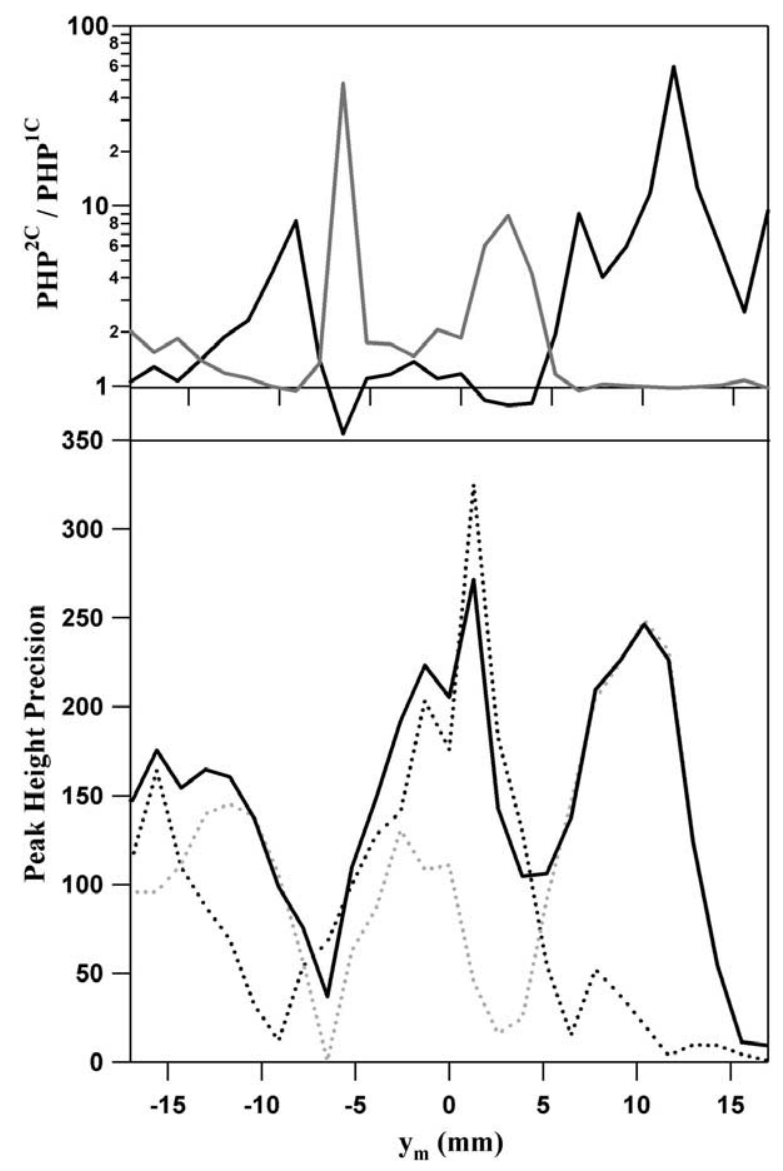

Figure 10. Two-channel advantage of peak height precision. The experimental peak heights and variances from Figure 9 were used to calculate the PHPs of the inner (black dotted line) and outer (gray dotted line) channels. The two spectra were summed to get the two-channel mass spectra. The PHP values of the summed peaks are also plotted (black solid line). On the top of the plot are the ratios of the two-channel PHP to the PHPs of the inner (black line) and the outer (gray line) channels. 


$$
\begin{aligned}
\mathrm{PHP}_{i}^{2 \mathrm{C}} & =\frac{F_{i}\left(\varepsilon^{\text {in }} f_{\mathrm{HT}}^{\text {in }}+\varepsilon^{\text {out }} f_{\mathrm{HT}}^{\text {out }}\right) \Psi_{\mathrm{Tot}}}{\sqrt{\left(f_{\mathrm{HT}}^{\text {in }}+f_{\mathrm{HT}}^{\text {out }}\right) \Psi_{\mathrm{Tot}}}} \\
& \approx F_{i} \varepsilon \sqrt{\left(f_{\mathrm{HT}}^{\text {in }}+f_{\mathrm{HT}}^{\text {out }}\right) \Psi_{\mathrm{Tot}}}
\end{aligned}
$$

The second approximation holds when $\varepsilon^{\text {in }}$ and $\varepsilon^{\text {out }}$ are equal. The ratio of PHPs for two-channel to one-channel is

$$
\frac{\mathrm{PHP}_{i}^{2 \mathrm{C}}}{\mathrm{PHP}_{i}^{\text {in }}}=\left(1+\frac{\varepsilon^{\text {out }}}{\varepsilon^{\text {in }}} \frac{f_{\mathrm{HT}}^{\text {out }}}{f_{\mathrm{HT}}^{\text {in }}}\right) / \sqrt{1+\frac{f_{\mathrm{HT}}^{\text {out }}}{f_{\mathrm{HT}}^{\text {in }}}} \approx \sqrt{\frac{f_{\mathrm{HT}}^{\text {in }}+f_{\mathrm{HT}}^{\text {out }}}{f_{\mathrm{HT}}^{\text {in }}}}
$$

This two-channel advantage mainly derives from the increased duty cycle if the modulation efficiencies of both channels are similar $\left(\varepsilon^{\text {in }} \approx \varepsilon^{\text {out }}\right)$, and it becomes $\sqrt{2}$ if the duty cycles are also equal $\left(f_{\mathrm{HT}}^{\text {in }}=f_{\mathrm{HT}}^{\text {out }}\right)$. However, if $\varepsilon^{\text {out }}$ is smaller than $\varepsilon^{\text {in }}$, this advantage is degraded and, conversely, if $\varepsilon^{\text {out }}$ is greater than $\varepsilon^{\text {in }}$, this advantage will exceed the ratio of duty cycles.

Figure 10 shows the peak height precision of the inner anode (black dotted line), the outer anode (gray dotted line), and the sum of the two (black solid line) for the experiments in Figure 9. At the top, the two-channel advantage is plotted for both channels. The two-channel PHP follows the maximum of the two one-channel PHPs. When PHP in is much smaller than PHPout, twochannel advantage of the inner channel is huge. However, when PHP ${ }^{\text {in }}$ is much larger than PHPout, the two-channel advantage can be smaller than 1 . When both single-channel PHPs are high, the two-channel advantage is approximately $\sqrt{2}$. Therefore, the duty cycles and modulation efficiencies of both channels should be maximized to achieve the best peak height precision.

To summarize, we have derived analytical expressions that accurately describe the ion deflection by the BNG. From these expressions and by integration over the ion beam distribution, ion counts of the two channels were calculated analytically. The calculated ion counts reflect the dynamic behavior by the BNG, ion beam distribution, and the detector sizes. The duty cycle and modulation efficiency contribute to the peak height and are good figures of merit for probing the performance of HT-TOFMS. The duty cycle and the ion modulation efficiency can be calculated from the theoretical ion counts and can be utilized for understanding and finding the optimum conditions of 2C-HT-TOFMS. Therefore, the modulation efficiency needs to be maximized by carefully matching the ion beam dimension, ion beam deflection, and the detector dimensions to exploit the $100 \%$ duty cycle and to achieve the best performance of the 2C-HT-TOFMS instrument.

\section{Acknowledgments}

This work was supported by the United States Air Force Office of Scientific Research (AFOSR grant FA9550-04-1-0076).

\section{References}

1. Zare, R. N.; Fernandez, F. M.; Kimmel, J. R. High-Speed Mass Spectrometry Hadamard Transform Time-of-Flight Mass Spectrometry: More Signal, More of the Time. Angew. Chem. Int. Ed. 2003, 42, 30-35

2. Trapp, O.; Kimmel, J. R.; Yoon, O. K.; Zuleta, I. A.; Fernandez, F. M.; Zare, R. N. Continuous Two-Channel Time-of-Flight Mass Spectrometric Detection of Electrosprayed Ions. Angew. Chem. Int. Ed. 2004, 43, 6541-6544

3. Guilhaus, M.; Selby, D.; Mlynski, V. Orthogonal Acceleration Time-of-Flight Mass Spectrometry. Mass Spectrom. Rev. 2000, 19, 65-107.

4. Verentchikov, A. N.; Ens, W.; Standing, K. G. Reflecting Time-of-Flight Mass Spectrometer with an Electrospray Ion Source and Orthogonal Extraction. Anal. Chem. 1994, 66, 126-133.

5. Kimmel, J. R.; Yoon, O. K.; Zuleta, I. A.; Trapp, O.; Zare, R. N. Peak Height Precision in Hadamard Transform Time-of-Flight Mass Spectra. J. Am. Soc. Mass Spectrom. 2005, 16, 1117-1130.

6. Bradbury, N. E.; Nielsen, R. A. Absolute Values of the Electron Mobility in Hydrogen. Phys. Rev. 1936, 49, 388-393.

7. Kimmel, J. R.; Engelke, F.; Zare, R. N. Novel Method for the Production of Finely Spaced Bradbury-Nielson Gates. Rev. Sci. Instrum. 2001, 72, 4354-4357.

8. Harwit, M. D.; Sloane, N. J. Hadamard Transform Optics; Academic Press: London, 1979, pp. 55-61.

9. Trapp, O.; Pearce, E. W.; Kimmel, J. R.; Yoon, O. K.; Zuleta, I. A.; Zare, R. N. A. Soft On-Column Metal Coating Procedure for Robust Sheathless Electrospray Emitters Used in Capillary Electrophoresis/Mass Spectrometry. Electrophoresis 2005, 26, 1358-1365.

10. Bethe, H. Über den Durchgang von Kathodenstrahlen durch Gitterförmige Elektrische Felder. Z. Phys. 1929, 54, 703-710

11. Yang, Z. Performance Advantages of Maximum Likelihood Methods in PRBS-Modulated Time-of-Flight Electron Energy Loss; Ph.D. Thesis, University of Maine, Orono, ME, 2003, pp. 37-39.

12. Sedlacek, M. Electron Physics of Vacuum and Gaseous Devices; Wiley and Sons: New York, 1996, p. 103.

13. Dahl, D. A. SIMION for the Personal Computer in Reflection. Int. J. Mass Spectrom. 2000, 200, 3-25.

14. Brock, A.; Rodriguez, N.; Zare, R. N. Characterization of a Hadamard Transform Time-of-Flight Mass Spectrometer. Rev. Sci. Instrum. 2000, 71, 1306-1318.

15. Rodriguez, N. Hadamard Transform Time-of-Flight Mass Spectrometry: Implementation and Characteristics; Ph.D. Thesis, Stanford University, Stanford, CA, 1999. 